\title{
Paediatric inflammatory bowel disease in a multiracial Asian country
}

Hui Ping $\underline{C h u}^{1}$, MBBS, MRCPCH, Veena Logarajah ${ }^{2}$, MBChB, MRCPCH, Nancy $\underline{T a n}^{2}$, MBBs, MRCPCH, Kong Boo $\underline{\text { Phua }}^{2}$, MBBS, MMed

INTRODUCTION This study examined the characteristics and trends in the incidence of inflammatory bowel disease (IBD) among children in Singapore.

METHODS We retrospectively reviewed all patients under 18 years diagnosed with IBD over a 14-year period. Information on demographics, disease presentation, laboratory findings, radiological investigations, and endoscopic and histological findings were obtained from the patients' medical records.

RESULTS 32 patients were diagnosed with IBD, 30 of whom had Crohn's disease and 2 had ulcerative colitis. The incidence of IBD rose from an initial rate of 2.2 per 100,000 patients in the year 2000 to a peak of 11.4 patients per 100,000 patients by 2008 . Median age of onset of symptoms was 10.5 years. There were more boys (63\%) than girls in the group and a higher representation of Indians (34.4\%). The most common presenting symptoms were abdominal pain (87.5\%), diarrhoea (75.0\%) and weight loss (71.9\%). Extraintestinal manifestations such as fever and arthralgia were found in over $50 \%$ of patients. The most common physical findings were perianal abnormalities (56.3\%), mouth ulcers $(37.5 \%)$ and growth failure (15.6\%). Abnormal laboratory findings such as low albumin, raised erythrocyte sedimentation rate, anaemia, thrombocytosis and high C-reactive protein were found in nearly half of the patients. Endoscopic and histological findings showed that a majority of patients $(90.6 \%)$ also had evidence of inflammation in the upper gastrointestinal tract.

CONCLUSION Paediatric IBD is on the rise. The higher occurrence in Indians, earlier onset and more florid presentation may suggest different genetic and environmental influences specific to Asian children.

Keywords: Asian races, Crohn's disease, paediatric IBD, ulcerative colitis

\section{INTRODUCTION}

Inflammatory bowel disease (IBD) was previously viewed as a condition that was more common in the West rather than in Asia. ${ }^{(1-3)}$ However, in recent years, the prevalence of IBD has been rising progressively in Asian countries. ${ }^{(4-6)}$ While this may be due to increased disease awareness and better diagnostic practices, it is also probably related to the Westernisation of lifestyle and diet, $^{(7)}$ which may be a leading cause of this increase in incidence. The precise aetiology of IBD is not known, but it is postulated to be a complex interaction between genetic predisposition, immune dysfunction and environmental triggers. ${ }^{(8)} 25 \%$ of all patients with IBD present in childhood, ${ }^{(9)}$ and paediatric patients with IBD have certain unique features such as growth failure, ${ }^{(10)}$ pan-colonic involvement ${ }^{(11)}$ and genetic mutations, particularly in early-onset disease. ${ }^{(12,13)}$ Hence, early diagnosis in the paediatric population is crucial so that prompt treatment can be initiated.

Singapore is a Southeast Asian city-state with an ethnically heterogeneous population of about five million, comprising Chinese $(74.1 \%)$, Malay (13.4\%), Indian (9.2\%) and other ethnic (3.3\%) races. ${ }^{(14)}$ In Singapore, the overall adult prevalence of ulcerative colitis (UC) is estimated to be 6 per 100,000 and that of Crohn's disease (CD) is around 3.6 per 100,000, ${ }^{(15)}$ with a greater predisposition among Indians for UC, although no such racial differences have been found for $\mathrm{CD}$. Limited data on
IBD in children has been published in Singapore. In this study, we examined the characteristics and trends in the incidence of paediatric IBD in Singapore over a 14-year period. Data were retrospectively reviewed to identify any differences in racial predisposition and manifestations of the disease in the local population when compared to paediatric patients with IBD in Western countries.

\section{METHODS}

We retrospectively reviewed all patients below 18 years of age who either attended outpatient clinics or were admitted to KK Women's and Children's Hospital (KKH), Singapore, and were diagnosed with IBD from 1996 to 2009. Data retrieved from the patients' medical records included demographic characteristics (such as age, gender and ethnicity), disease presentation (including extraintestinal manifestations), anthropometric measurements, as well as findings of physical examination, laboratory investigations, radiographic evaluations, and endoscopic and histological assessments.

The diagnosis of IBD was established based on clinical history, physical examination, and radiographic, endoscopic and histopathological evidence. A diagnosis of $C D$ was made when one or more of the following was present: (a) a granuloma in either an upper or lower gastrointestinal biopsy, using

${ }^{1}$ Raffles Children's Centre, Raffles Hospital, ${ }^{2}$ Gastroenterology Service, Department of Paediatric Medicine, KK Women's and Children's Hospital, Singapore Correspondence: Dr Chu Hui Ping, Consultant Paediatrician, Raffles Children's Centre, 585 North Bridge Road, \#12-00 Raffles Hospital, Singapore 188770. chu_huiping@rafflesmedical.com 
endoscopy; (b) endoscopic features (such as aphthous or linear ulcers, cobblestone appearance, and skip/discontinuous lesions); (c) perianal disease (such as fistulae, large skin tags or abscesses); (d) transmural inflammation (such as stricturing or fistulising disease); and/or (e) radiological changes in the small intestine consistent with CD. A diagnosis of UC was made in the presence of both: (a) inflammatory changes (including superficial ulcerations, granularity and/or friability with crypt abscesses); and (b) exclusive colonic involvement and continuous distribution extending proximally from the rectum. Stool cultures were also performed to exclude bacterial infection. Exceptions that did not change the diagnosis of UC included nonspecific upper gastrointestinal inflammation and pancolitis with backwash ileitis, which were not considered to be extracolonic involvement.

The incidence rates of $C D$ and UC were calculated using the number of new patients diagnosed per 100,000 patients seen in a particular year. KKH is one of two public children's hospitals in Singapore that provides both ambulatory and inpatient healthcare services for children and attends to the needs of almost 80,000 paediatric patients each year.

\section{RESULTS}

Over a period of 14 years, a total of 32 patients were diagnosed with IBD. Of these, 30 (94\%) patients had CD and 2 (6\%) had UC. The diagnoses of two patients who were previously labelled as indeterminate colitis at initial/first presentation were subsequently changed to $C D$ and $U C$, respectively, on repeat endoscopy. The incidence of IBD was found to have increased over the years, from 0-1 new patient being diagnosed per year in the late 1990s to a maximum of eight patients being diagnosed in 2008 (Fig. 1). The respective annual incidence rates of IBD reflected this rise (Fig. 2), from an incidence of 2.2 per 100,000 patients in 2000 to the highest incidence of 11.4 patients per 100,000 patients in 2008 .

There was a male preponderance $(63 \%)$ among patients with $\mathrm{CD}$, with a male-to-female ratio of 1.7:1. The gender ratio was equal for patients with UC. The median age of patients at presentation was 10.8 (range 5-17) years. The age distribution of patients at presentation showed two peaks - age groups 8 to $<10$ years and 12 to $<14$ years (Fig. 3). The racial distribution of patients with IBD was $16(50.0 \%)$ Chinese, 2 (6.3\%) Malays, 11 (34.4\%) Indians and 3 (9.4\%) patients of other minor ethnic groups (Indian-Chinese $\mathrm{n}=1$; Afro-Indian $\mathrm{n}=1$; German-Thai $n=1$ ) (Table I). The two patients with UC were of non-Chinese ethnicity (Malay $\mathrm{n}=1$; Indian $\mathrm{n}=1$ ). None of the patients had a family history of IBD.

The most common presenting symptoms were abdominal pain $(87.5 \%)$, diarrhoea $(75.0 \%)$ and weight loss $(71.8 \%)$. $16(50.0 \%)$ patients had all the above three symptoms at the initial presentation. Extraintestinal manifestations, mainly in the form of intermittent fever and arthralgia, were seen in 17 (53.1\%) patients. The most common physical findings were perianal abnormalities (56.3\%), such as skin tags, fissures and fistulae, aphthous ulcers in

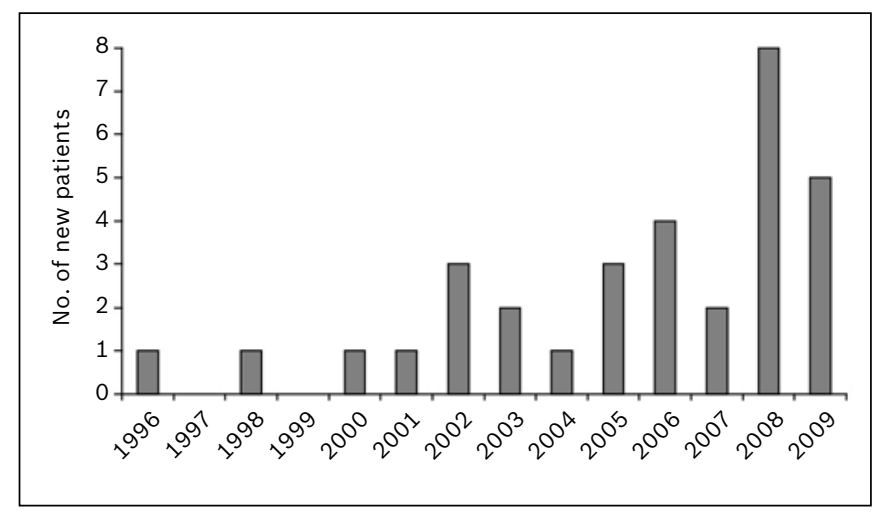

Fig. 1 Graph shows the number of newly diagnosed patients with inflammatory bowel disease per year.

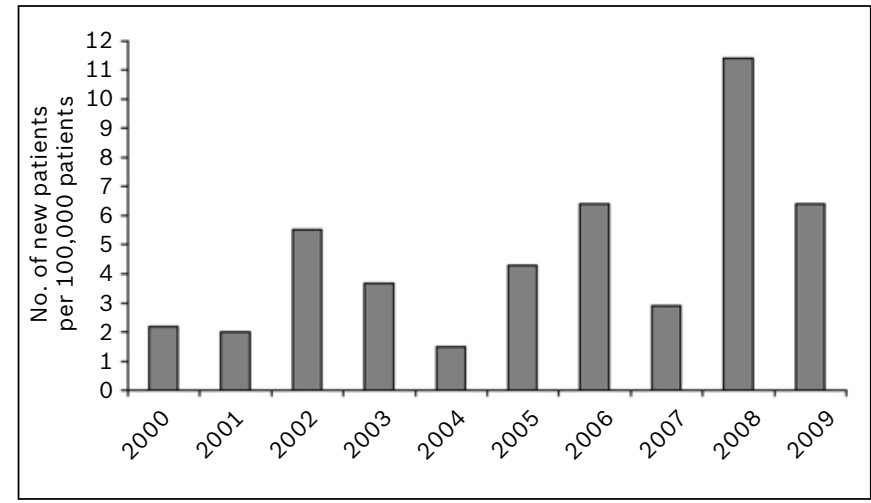

Fig. 2 Graph shows the annual incidence rates (number of new patients per 100,000 patients per year) of inflammatory bowel disease.

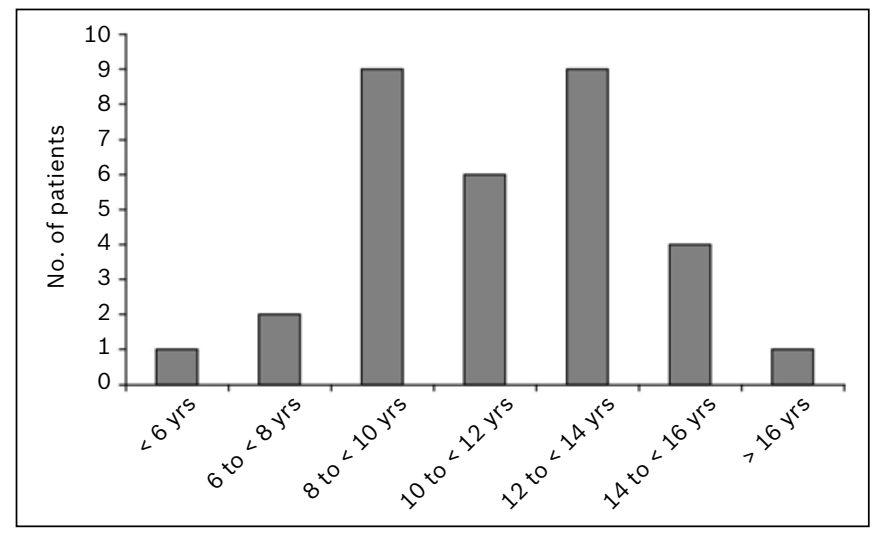

Fig. 3 Distribution by age group at the time of first presentation of inflammatory bowel disease.

the mouth (37.5\%) and growth failure (15.6\%), which was defined as weight and/or height at the third percentile or below for the patient's age. However, no abnormal physical findings were found in $10(31.3 \%)$ patients at the initial presentation (Table I).

Initial laboratory investigations revealed low albumin, raised erythrocyte sedimentation rate (ESR), anaemia, thrombocytosis and high C-reactive protein (CRP) in more than $50 \%$ of patients with IBD (Table I). A majority of patients $(90.6 \%)$ had at least three of the above five abnormal laboratory findings at the time of their initial presentation. Five patients among the seven patients who underwent barium follow-through studies had ulcers in the terminal ileum. Among the ten patients who had ultrasonography of the abdomen, bowel wall thickening was found in five patients 
Table I. Patient characteristics $(n=32)$.

\begin{tabular}{|c|c|}
\hline Characteristic & No. (\%) \\
\hline \multicolumn{2}{|l|}{ Ethnicity } \\
\hline Chinese & $16(50.0)$ \\
\hline Malay & $2(6.3)$ \\
\hline Indian & $11(34.4)$ \\
\hline Others & $3(9.4)$ \\
\hline \multicolumn{2}{|l|}{ Presenting symptom } \\
\hline Abdominal pain & $28(87.5)$ \\
\hline Diarrhoea & $24(75.0)$ \\
\hline Weight loss & $23(71.9)$ \\
\hline Blood in stool & $17(53.1)$ \\
\hline Loss of appetite & $15(46.9)$ \\
\hline Intermittent fever & $14(43.8)$ \\
\hline Mouth ulcers & $14(43.8)$ \\
\hline Nocturnal diarrhoea & $5(15.6)$ \\
\hline Arthralgia & $5(15.6)$ \\
\hline Mucus in stools & $4(12.5)$ \\
\hline Tenesmus & $3(9.4)$ \\
\hline \multicolumn{2}{|l|}{ Physical finding } \\
\hline Aphthous ulcer & $12(37.5)$ \\
\hline Perianal skin tag & $10(31.3)$ \\
\hline Perianal fissure & 9 (28.1) \\
\hline Perianal fistula & $5(15.6)$ \\
\hline Growth failure & $5(15.6)$ \\
\hline Arthritis & $3(9.4)$ \\
\hline Delayed puberty & $2(6.3)$ \\
\hline No abnormal findings & $10(31.3)$ \\
\hline \multicolumn{2}{|l|}{ Abnormal laboratory finding } \\
\hline Low albumin & $28(87.5)$ \\
\hline Raised ESR & $26(81.3)$ \\
\hline Anaemia & $25(78.1)$ \\
\hline Thrombocytosis & $23(71.9)$ \\
\hline High CRP & $16(50.0)$ \\
\hline Elevated WBC count & 15 (46.9) \\
\hline Positive stool occult blood & $11(34.4)$ \\
\hline Positive ANCA & $9(28.1)$ \\
\hline Raised liver enzyme & $2(6.3)$ \\
\hline Raised ALP/GGT & $2(6.3)$ \\
\hline
\end{tabular}

ALP: alkaline phosphatase; ANCA: antineutrophil cytoplasmic antibody; CRP: C-reactive protein; ESR: erythrocyte sedimentation rate; GGT: gammaglutamyl transpeptidase; WBC: white blood cell

and lympahdenopathy was seen in three patients. Among the five patients who underwent computed tomography, three had bowel wall thickening in the colon, one had thickening of the terminal ileum and one had lymphadenopathy alone.

All 32 patients underwent colonoscopy. The two patients with UC had continuous pan-colonic involvement, with features of superficial ulcerations, mucosal erythema and friability. Among the 30 patients with CD, 24 had skip or discontinuous lesions and 5 had pan-colonic mucosal abnormality. The one patient who had normal colonoscopy findings was diagnosed with $\mathrm{CD}$ only when multiple jejunal and ileal ulcers were found on video capsule endoscopy and barium follow-through studies. 27 patients with CD had aphthous or linear ulcers on colonoscopy. Among the 27 IBD patients who also underwent upper gastrointestinal endoscopy, macroscopic gastritis was detected in the two patients with $\cup C$, isolated erythema of the lower oesophagus and/or stomach in three patients with CD, and aphthous ulcers of either the lower oesophagus, stomach or duodenum in seven patients with CD. On histology, 15 patients were positive for granulomas on biopsy and eight patients had definite active ileitis. Inflammation of the upper gastrointestinal tract was seen in 25 patients ( $2 \mathrm{UC}$ and $23 \mathrm{CD}$ patients). Ten patients with $\mathrm{CD}$ and one patient with $\cup \mathrm{C}$ also had Helicobacter pylori colonisation. Five patients with $\mathrm{CD}$ and one patient with UC were given triple therapy due to the presence of dyspeptic symptoms and/or findings of active gastritis on histology.

\section{DISCUSSION}

Our series of 32 paediatric patients is the first study of its kind examining the characteristics and incidence of childhood IBD in Singapore. The incidence of paediatric IBD in the United States and Europe ranges from 0.7 to 8.4 per 100,000 children for CD and 0.5 to 3.3 per 100,000 children for UC. ${ }^{(16-19)}$ In comparison, there is a paucity of data on IBD in Asian children. In Japan, the proportion of newly diagnosed paediatric patients registered with a nationwide IBD registry was $10.6 \%$ for CD and $5.9 \%$ for UC. ${ }^{(20)}$ In South India, paediatric patients accounted for $7 \%$ of new patients with IBD seen annually. ${ }^{(21)}$ In Taiwan, only ten children were diagnosed with CD over a period of five years in a tertiary medical referral centre that has an annual admission of approximately 7,000 patients. ${ }^{(22)}$ In Singapore, the prevalence of adult CD was previously estimated to be around 3.6 per 100,000 , while that of UC was reportedly 6 per 100,000 in the year 2000. ${ }^{(15)}$ However, there is limited information on childhood IBD in Singapore. Only one local study in 1992 reported six cases of paediatric IBD over a duration of 21 months - four patients had UC, one had CD and one had nonspecific colitis; thus, the estimated national incidence of paediatric IBD was $1-2$ per 100,000 . $^{(23)}$

Although we were unable to calculate the true incidence of paediatric IBD for Singapore as a whole from our study, the hospital-based incidence rates from our data could be construed as a reliable indicator of its trend, as $\mathrm{KKH}$ is the largest tertiary medical centre for children in the country. Our findings indicate that the annual number of newly diagnosed paediatric patients with IBD has tripled over the study period, from 2.2 per 100,000 patients in the year 2000 to 6.4 per 100,000 patients in 2009. This rising trend over the last ten years is in keeping with the reported global rise in the incidence of paediatric IBD. ${ }^{(24)}$ There could be multiple reasons behind this increase. The spike may in part be due to an increased awareness among the general public as well as the availability of better diagnostic techniques. The Westernisation of everyday life in Asia may be another likely contributing factor. ${ }^{(7)}$ While the genetic differences between Asian and Western children may have accounted for the much lower prevalence of IBD noted among Asians previously, the ever-increasing Western influences on lifestyle and dietary habits might be contributing to the fast-rising incidence of IBD in Asian countries today. This emphasises the fact that IBD is a multifactorial disease, with a complex interplay of genetic predisposition and environmental triggers that may determine the likely course of disease.

A larger proportion of children in our study had CD compared to $\mathrm{UC}$, which is consistent with the distribution of 
paediatric IBD reported in Western countries. ${ }^{(16-19)}$ However, our findings are contrary to previous studies which reported that UC was more predominant in Asian children than CD. ${ }^{(25)}$ It is possible that these earlier reports extrapolated to paediatric patients the interpretations of other studies that had found a higher prevalence of UC among Asian adults. ${ }^{(15,26,27)}$ However, the proportion of patients with CD and UC in our study (15:1) was much higher than that in the United Kingdom (2:1), indicating perhaps that paediatric patients with UC were under-represented in our study. ${ }^{(25)}$ It is possible that some of our patients with UC were misdiagnosed and treated for infective diarrhoea, and thus were not referred for further workup or endoscopy. However, it is unlikely that the number of such missed patients was very high, as the study's surveillance period was 14 years long and any missed patients would have been picked up when they presented again with symptoms.

None of our patients had a positive family history of IBD, which contrasted with paediatric populations in the West where a strong association with family history (up to $42 \%$ ) was seen. ${ }^{(28)}$ Hsiao et al, who looked for common mutations in the NOD2/CARD15 and TLR4 genes, which are strongly associated with $\mathrm{CD}$ among Caucasian patients, was unable to find any such mutations in Taiwanese children with CD. ${ }^{(22)}$ This may indicate the existence of different genetic influences that are specific to the development of IBD in Asian children.

Strikingly, a higher representation of Indians was seen among our patients with $C D$, with patients of Indian ethnicity making up over a third of the patients, despite the fact that Indians account for less than $10 \%$ of the entire local population. As the number of patients with UC in our study was small, we were unable to establish the racial distribution of these patients. Our findings differ from previous reports of adult patients from Singapore ${ }^{(15)}$ and Malaysia, ${ }^{(29)}$ where a higher prevalence for UC but not for CD was found among Indians. There was also a higher representation of other minor ethnic groups among our patients with IBD. The authors expect the numbers associated with other ethnicities to increase in the future as immigration continues to rise and interracial marriages become more common.

The overall clinical presentation of IBD in our Asian cohort is similar to that seen among paediatric patients with IBD in Western populations. There was a higher prevalence of boys with $C D$ in our study, which is consistent with the male predisposition to IBD reported in several studies. ${ }^{(25,30)}$ Abdominal pain, the top reported symptom among children with IBD, was also the most frequently seen symptom in our series. ${ }^{(11)}$ This is different from adult patients with IBD, who usually present with either rectal bleeding (in patients with $U C$ ) or diarrhoea (in those with $\mathrm{CD}$ ). Growth failure was another common feature in our patients $(15.6 \%)$, which is in keeping with previous studies that have reported growth failure in $10 \%-40 \%$ of children with IBD. ${ }^{(10)}$

However, a few differences have been noted between our study findings and those of other studies. The median age of patients at initial presentation was 10.5 years in our series, which is much lower than that reported in the United States and United Kingdom (average age, 12-13 years). ${ }^{(11,25,31)}$ The classic triad of symptoms abdominal pain, diarrhoea and weight loss - was found in half of our patients, which is also much higher compared to British children with IBD (25\%). ${ }^{(11)}$ Extraintestinal manifestations, in the form of intermittent fever and arthralgia, were more frequently present in our series $(53.1 \%)$ when compared to studies from the United States and Europe (range 10\%-36\%). ${ }^{25,32,33)}$ Our findings seem to imply that IBD in Asian children has an earlier onset and a more florid presentation of gastrointestinal and systemic symptoms.

In terms of anatomical distribution of the disease, the two patients with UC in our study had pan-colitis, which is the most common phenotype reported in children with UC. ${ }^{(11)}$ We were unable to confidently categorise our patients with $\mathrm{CD}$ according to the site of disease activity (such as ileitis, colitis or ileocolitis) as the data on ileum intubation during endoscopy and small bowel imaging were scanty. Only 11 patients with CD had proven ileal involvement based on either biopsy results or radiological imaging (such as barium follow-through studies or computed tomography). However, the authors are of the opinion that the sites of disease involvement in our patients were similar to that in other children with $\mathrm{CD}$, with ileocolonic involvement being the most common type. ${ }^{(11)} \mathrm{A}$ majority of our patients had positive findings on colonoscopy and a large proportion had abnormal laboratory results such as anaemia and hypoalbuminaemia, which are suggestive of small intestinal involvement. Only one patient had isolated small bowel $C D$, which is reportedly rare in children with CD (9\%). ${ }^{(11)}$ The high frequency of occurrence of upper gastrointestinal inflammation in our series is also consistent with that of previous reports (range $50 \%-64 \%$ ). ${ }^{(34,35)}$ These findings emphasise the importance of routine upper endoscopy in all children suspected of having IBD regardless of any upper gastrointestinal symptoms, as findings of aphthous ulcers or granulomas may help to confirm a diagnosis of $C D$, which might otherwise be missed. The high incidence of $H$. pylori colonisation in our patients was likely related to its high prevalence in the local population. ${ }^{(36)}$ It would, however, be interesting to evaluate the role of $H$. pylori in the development of IBD since it has been postulated to have a protective effect due to its ability to induce immune tolerance and limit inflammatory responses. ${ }^{(37)}$

In conclusion, IBD in Asian children is an emerging disease. Its rising incidence may be associated with a number of factors, such as increased awareness, improved diagnostic investigations, the rapid Westernisation of societies and progress on the socioeconomic development front. The apparent earlier onset and more florid presentation among Asian paediatric patients with IBD may imply a different set of genetic and environmental influences from that reported among children with IBD in Western countries. More epidemiological data and genetic studies specific to Asian countries are required so as to better understand and manage this condition. 


\section{REFERENCES}

1. Tan CC, Kang JY, Guan R, Yap I, Tay HH. Inflammatory bowel disease: an uncommon problem in Singapore. J Gastroenterol Hepatol 1992; 7:360-2.

2. Thein-Htut, Kudva MV. Ulcerative colitis in Malaysians: a review of 23 patients. Singapore Med J 1989; 30:385-7.

3. Probert CS, Mayberry JF, Mann R. Inflammatory bowel disease in the rural Indian subcontinent: a survey of patients attending mission hospitals. Digestion 1990; 47:42-6.

4. Sood A, Midha V. Epidemiology of inflammatory bowel disease in Asia. Indian J Gastroenterol 2007; 26:285-9.

5. Lakatos PL. Recent trends in the epidemiology of inflammatory bowel disease: up or down? World J Gastroenterol 2006 14; 12:6102-8.

6. Kitahora T, Utsunomiya T, Yokota A. Epidemiological study of ulcerative colitis in Japan: incidence and familial occurrence: The Epidemiology Group of the Research Committee of Inflammatory Bowel disease in Japan. J Gastroenterol 1995; 30:5-8.

7. Thia KT, Loftus EV Jr, Sandborn WJ, Yang SK. An update on the epidemiology of inflammatory bowel disease in Asia. Am J Gastroenterol 2008; 103:3167-82.

8. Beattie M. Inflammatory bowel disease: introduction. In: Beattie M, Dhawan A, Puntis JWL. Paediatric gastroenterology, hepatology, and nutrition. New York: Oxford University Press, 2009:287-90.

9. Mamula P, Markowitz JE, Baldassano RN. Inflammatory bowel disease in early childhood and adolescence: special considerations. Gastroenterol Clin North Am 2003; 32:967-95.

10. Motil KJ, Grand RJ, Davis-Kraft L, Ferlic LL, Smith EO. Growth failure in children with inflammatory bowel disease: a prospective study. Gastroenterology 1993; 105:681-91.

11. Sawczenko A, Sandhu BK. Presenting features of inflammatory bowel disease in Great Britain and Ireland. Arch Dis Child 2003; 88:995-1000.

12. Sun L, Roesler J, Rosen-Wolff A, et al. CARD15 genotype and phenotype analysis in 55 paediatric patients with Crohn disease from Saxony, Germany. J Pediatr Gastroenterol Nutr 2003; 37:492-7.

13. Sanchez R, Levy E, Costea F, Sinnett D. IL-10 and TNF promoter haplotypes are associated with childhood Crohn's disease location. World J Gastrenterol 2009; 15:3776-82.

14. Singapore Economic Development Board. Singapore Facts \& Figures [online].http://www.edbsingapore.jp/edb/sg/en_uk/index/why_singapore/ singapore_facts_and_figures.html\#people. Accessed February 28, 2013.

15. Lee YM, Fock K, See SJ, et al. Racial differences in the prevalence of ulcerative colitis and Crohn's disease in Singapore. J Gastroenterol Hepatol 2000; 15:622-5.

16. Barton JR, Gillon S, Ferguson A. Incidence of inflammatory bowel disease in Scottish children between 1968 and 1983; marginal fall in ulcerative colitis, three-fold rise in Crohn's disease. Gut 1989; 30:618-22.

17. Hildebrand $\mathrm{H}$, Finkel $\mathrm{Y}$, Grahnquist $\mathrm{L}$, et al. Changing pattern of paediatric inflammatory bowel disease in northern Stockholm 1990-2001. Gut 2003; 52:1432-4

18. Sawczenko A, Sandhu BK, Logan RF, et al. Prospective survey of childhood inflammatory bowel disease in the British Isles. Lancet 2001; 357:1093-4.

19. Kugathasan S, Judd RH, Hoffmann RG, et al. Epidemiologic and clinical characteristics of children with newly diagnosed inflammatory bowel disease in Wisconsin: a statewide population-based study. J Pediatr 2003; 143:525-31.

20. Ishige T, Tomomasa T, Takebayashi T, et al. Inflammatory bowel disease in children: epidemiological analysis of the nationwide IBD registry in Japan. J Gastroenterol 2010; 45:911-7.

21. Avinash B, Dutta AK, Chacko A. Pediatric inflammatory bowel disease in South India. Indian Pediatr 2009; 46:639-40.

22. Hsiao $\mathrm{CH}$, Wei SC, Wong JM, et al. Pediatric Crohn disease: clinical and genetic characteristics in Taiwan. J Pediatr Gastroenterol Nutr 2007, 44:342-6.

23. Tan A, Wee A, Quak SH. Chronic inflammatory bowel disease in Singapore children. J Singapore Paediatr Soc 1992; 34:209-13.

24.IBD working group of the European Society for Paediatric Gastroenterology, Hepatology and Nutrition. Inflammatory bowel disease in children and adolescents: recommendations for diagnosis - the Porto criteria. J Pediatr Gastroenterol Nutr 2005; 41:1-7.

25. UK IBD working group on behalf of the British Society of Paediatric Gastroenterology Hepatology and Nutrition (BSPGHAN). Guidelines for the management of inflammatory bowel disease (IBD) in children in the United Kingdom. In: British Society of Paediatric Gastroenterology Hepatology and Nutrition [online]. Available at: www.bspghan.org.uk/ working_groups/documents/IBDGuidelines_000.pdf. Accessed October 19, 2010.

26. Yang SK, Yun S, Kim JH, et al. Epidemiology of inflammatory bowel disease in the Songpa-Kangdong district, Seoul, Korea, 1986-2005: a KASID study. Inflamm Bowel Dis 2008; 14:542-9.

27. Jiang L, Xia B, Li J, et al. Retrospective survey of 452 patients with inflammatory bowel disease in Wuhan city, central China. Inflamm Bowel Dis $2006 ; 12: 212-7$

28. Weinstein TA, Levine $M$, Pettei MJ, et al. Age and family history at presentation of pediatric inflammatory bowel disease. J Pediatr Gastroenterol Nutr 2003; 37:609-13.

29. Tan YM, Goh KL. Ulcerative colitis in a multiracial Asian country: racial differences and clinical presentation among Malaysian patients. World Gastroenterol 2005; 11:5859-62.

30. Lindberg E, Lindquist B, Holmquist L, Hildebrand H. Inflammatory bowel disease in children and adolescents in Sweden, 1984-1995. J Pediatr Gastroenterol Nutr 2000; 30:259-64.

31. Abramson O, Durant M, Mow W, et al. Incidence, prevalence, and time trends of pediatric inflammatory bowel disease in Northern California, 1996 to 2006. J Pediatr 2010; 157:233-9.

32. Su CG, Judge TA, Lichtenstein GR. Extraintestinal manifestations in inflammatory bowel disease. Gastroenterol Clin North Am 2002; 31:307-27.

33. Veloso FT, Carvalho J, Magro F. Immune-related systemic manifestations of inflammatory bowel disease. A prospective study of 792 patients. J Clin Gastroenterol 1996; 23:29-34.

34. Castellaneta SP, Afzal NA, Greenberg M, et al. Diagnostic role of upper gastrointestinal endoscopy in pediatric inflammatory bowel disease. J Pediatr Gastroenterol Nutr 2004; 39:257-61.

35. Abdullah BA, Gupta SK, Croffie JM, et al. The role of esophagogastroduodenoscopy in the initial evaluation of childhood inflammatory bowel disease: a 7-year study. J Pediatr Gastroenterol Nutr 2002; 35:636-40

36. Fock KM. Helicobacter pylori infection - current status in Singapore. Ann Acad Med Singapore 1997; 26:637-41.

37. Luther J, Dave M, Higgins PD, Kao JY. Association between Helicobacter pylori infection and inflammatory bowel disease: a meta-analysis and systematic review of the literature. Inflamm Bowel Dis 2010; 16:1077-84. 